\title{
The role of profilin-1 in endothelial cell injury induced by advanced glycation end products (AGEs)
}

Zhenyu Li $i^{1}$, Qiaoging Zhong ${ }^{2}$, Tianlun Yang ${ }^{3}$, Xiumei Xie ${ }^{1}$ and Meifang Chen ${ }^{1 *}$

\begin{abstract}
Background: Accumulation of advanced glycation end products (AGEs) in the vasculature triggers a series of morphological and functional changes contributing to endothelial hyperpermeability. The reorganisation and redistribution of the cytoskeleton regulated by profilin-1 mediates endothelial cell contraction, which results in vascular hyperpermeability. This study aimed to investigate the pivotal role of profilin-1 in the process of endothelial cell damage induced by AGEs.
\end{abstract}

Methods: Human umbilical vein endothelial cells (HUVECs) were incubated with AGEs. The mRNA and protein expression of profilin-1 was determined using real-time PCR and western blotting analyses. The levels of intercellular adhesion molecule-1 (ICAM-1), nitric oxide (NO) and reactive oxygen species (ROS), as well as the activities of nuclear factor-KB (NF-KB) and protein kinase C (PKC), were detected using the appropriate kits. The levels of asymmetric dimethylarginine (ADMA) were determined using HPLC. The distribution of the cytoskeleton was visualised using immunofluorescent staining.

Results: Compared with the control, incubation of endothelial cells with AGEs $(200 \mu \mathrm{g} / \mathrm{ml})$ for 4 or $24 \mathrm{~h}$ significantly up-regulated the mRNA and protein expression of profilin-1, markedly increased the levels of ICAM-1 and ADMA and decreased the production of $\mathrm{NO}(P<0.05, P<0.01)$, which was significantly attenuated by pretreatment with DPI (an antioxidant), GF 109203X (PKC inhibitor) or BAY-117082 (NF-KB inhibitor). DPI (10 $\mu \mathrm{mol} / \mathrm{L})$ markedly decreased the

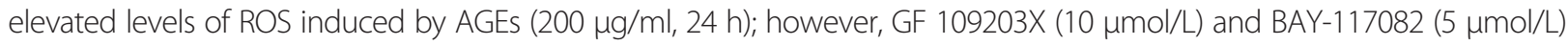
exhibited no significant effect on the formation of ROS by AGEs. Immunofluorescent staining indicated that AGEs markedly increased the expression of profilin- 1 in the cytoplasm and the formation of actin stress fibres, resulting in the rearrangement and redistribution of the cytoskeleton. This effect was significantly ameliorated by DPI, GF 109203X, BAY-117082 or siRNA treatment of profilin-1. Incubation with DPI and GF 109203X markedly inhibited the activation of PKC triggered by AGEs, and DPI and BAY-117082 significantly decreased the activity of NF-KB mediated by AGES. Disruption of profilin-1 gene expression attenuated the extent of endothelial abnormalities by reducing ICAM-1 and ADMA levels and elevating NO levels $(P<0.05, P<0.01)$, but this disruption had no effect on the activities of $N F-K B$ and PKC (P>0.05).

Conclusions: These findings suggested that profilin-1 might act as an ultimate and common cellular effector in the process of metabolic memory (endothelial abnormalities) mediated by AGEs via the ROS/PKC or ROS/NF-қB signalling pathways.

Keywords: Advanced glycation end products (AGEs), Profilin-1, Endothelial cells, Cytoskeletal rearrangement, Reactive oxygen species (ROS), Nuclear factor kappa B (NF-KB), Protein kinase C (PKC)

\footnotetext{
* Correspondence: chenmeifang16@hotmail.com

'Department of Geriatric Medicine, Xiang-Ya Hospital, Central South

University, Xiang-Ya Road 87\#, Changsha, Hunan 410008, China

Full list of author information is available at the end of the article
} 


\section{Background}

Diabetes mellitus (DM) is a serious and rapidly growing disease, and diabetes-related vascular complications are major causes of patient disability and death. Large-scale clinical studies have confirmed that early intensive blood glucose control can reduce the incidence of diabetic microvascular and macrovascular complications. However, for patients with chronic long-term hyperglycaemia, despite strict future long-term glycaemic control, diabetesrelated vascular complications remain or can develop. This phenomenon is known as "metabolic memory" or "hyperglycaemia memory." There is growing evidence that faster-generated advanced glycation end products (AGEs) in the conditions of long-term high glucose may be a unifying explanation for this phenomenon [1]. Previous studies have demonstrated that AGEs are involved in the pathogenesis of endothelial dysfunction in diabetic vascular complications, and its levels in diabetic patients are highly correlated with the severity of macrovascular and microvascular complications [2,3]. However, the mechanism of "metabolic memory" mediated by AGEs has not been fully elucidated to date. The direct effects of AGEs include protein glycation and crosslinking, which affect normal protein physiological functions. In addition, this process is prolonged and irreversible. There is a growing body of evidence that AGEs mediate "metabolic memory", primarily via indirect pathways via its receptors (receptor for advanced glycation end products, RAGE) [4]. Indeed, binding of AGEs to RAGE produces the excess formation of reactive oxidative species (ROS) independent of actual glucose, which subsequently activates protein kinase $\mathrm{C}$ (PKC) and the redox-sensitive transcription factor nuclear factor kappa B (NF-kB) via intracellular signalling cascade reactions. This activation subsequently initiates the expression of a variety of diabetes-related genes and RAGE [5]. Thus, self-maintaining conditions linked to AGE formation demonstrate that AGEs can conceivably contribute to "metabolic memory."

Profilin-1 as an actin-binding protein is a class of small molecule proteins (12 to $15 \mathrm{KD}$ ) and is widely distributed in various types of cells with highly conserved sequences. This plays an important role in the regulation of actin polymerisation in a number of motility functions. The reorganisation and redistribution of the cytoskeleton, particularly actin proteins, forms a pathological basis for endothelial cell contraction and increased vascular permeability, which contributes to endothelial abnormalities and vascular disease [6]. Under pathological conditions, such as diabetes or atherosclerosis (AS), profilin-1 levels were increased in atherosclerotic lesions, the aorta or in serum. It was recently reported that profilin-1 overexpression triggered indicators of endothelial dysfunction and attenuated the expression of profilin-1 conferred protection from AS in vivo $[7,8]$. In addition, Romeo et al. reported that profilin-1 and low density lipoprotein (LDL) was downstream molecules mediating diabetic endothelial dysfunction, and revealed that the endothelial damage triggered by the profilin-1 pathway in diabetes and in lipid oxidation was surprisingly similar $[7,9]$. Thus, we hypothesized that profilin-1 may be a common and ultimate pathway in endothelial cell injuries, and blockade of profilin-1-mediated biological effects may help to prevent the occurrence of endothelial abnormalities and vascular disease.

Recent studies have demonstrated that incubation of endothelial cells with AGEs caused a significant increase in endothelial permeability via cytoskeletal alterations and actin rearrangement $[10,11]$. Due to the pivotal role of profilin-1 in the reorganisation and redistribution of actin, we propose that profilin-1 may be involved in metabolic memory mediated by AGEs as an ultimate pathway in endothelial injury. Thus, the present study aimed to elucidate the role of profilin-1 in endothelial injury mediated by AGEs and its underlying signal transduction pathways.

\section{Materials and methods Chemicals and reagents}

Human umbilical vein endothelial cells (HUVEC12, ATCC,CRL-2480) were obtained from the Tumor Research Institute of Beijing Medical University (Beijing, China). Dulbecco's modified Eagle's medium (DMEM), Trizol reagents and Phallotoxins were obtained from Invitrogen. Foetal bovine serum (FBS) was supplied by Every Green Co. Ltd (Hangzhou, China). AGE-bovine serum albumin (AGE-BSA), diphenyliodonium (DPI), GF 109203X and BAY-117082 were purchase from Merck. Asymmetric dimethylarginine (ADMA) standard was purchased from Sigma. Intercellular adhesion molecule-1 (ICAM-1) ELISA kits and Griess reagents were purchased from Jiancheng Biological Medical Engineering Institute (Nanjing, China). ROS detection kits and BCA protein kits were purchased from Beyotime Company (Jiangsu, China). Rabbit anti-human-profilin1 antibody was purchased from Santa Cruz. Western blotting kits and secondary rat anti-rabbit IgG were purchased from KPL. First strand cDNA synthesis kit was obtained from Fermentas. The primers were synthesised by Takara (Dalian, China). TRIzol, the PepTag Assay for Non-Radioactive Detection of Protein Kinase C and pGL4.32[luc2P/NF-kB-RE/Hygro] Vector was obtained from Promega. pLNCX2-siprofilin-1 plasmids were synthesised by Clonetech and lipofectamine 2000 was supplied by Invitrogen.

\section{Cell culture}

HUVEC12 cells were maintained in DMEM containing $10 \%$ foetal bovine serum, $100 \mathrm{U} / \mathrm{ml}$ penicillin, and 100 $\mu \mathrm{g} / \mathrm{ml}$ streptomycin and grown in humidified atmosphere of $5 \% \mathrm{CO}_{2}$ in air at $37^{\circ} \mathrm{C}$. HUVEC12 cells were 
cultured in six-well plates at a density of $5 \times 10^{4}$ cells per well in DMEM until 70\%-80\% confluence. Each well was then washed twice with phosphate-buffered saline (PBS), and $2 \mathrm{ml}$ of DMEM containing various concentrations of AGEs $(100 \mu \mathrm{g} / \mathrm{ml}, 200 \mu \mathrm{g} / \mathrm{ml}, 400 \mu \mathrm{g} / \mathrm{ml})$ were added for different time periods $(0,6,12,24,48 \mathrm{~h})$. To elucidate the potential signal pathways, DPI (antioxidants, $10 \mu \mathrm{mol} / \mathrm{L}$ ), GF 109203X (PKC inhibitor, 10 $\mu \mathrm{mol} / \mathrm{L}$ ) and BAY-117082 (NF-kB inhibitor, $5 \mu \mathrm{mol} / \mathrm{L}$ ) were used in the study.

\section{RNA isolation and real-time PCR analysis}

Total RNA was extracted from cells grown in a 6-well plate using Trizol reagent and cDNA was synthesised from $1 \mu \mathrm{g}$ total RNA using the First-Strand Synthesis System for PCR according to the manufacturer's protocol. The primer pairs used in amplification of profilin-1 were: forward primer, 5' - CTGTCAGGACGCGGCCATCG -3'; reverse primer, 5 '-CAGCTGGCGTGATGTTGACGA-3'. The primer pairs of GAPDH were: forward primer, $5^{\prime}-\mathrm{GT}$ CGCCAGCCGAGCCACATC-3'; reverse primer, 5'-CC AG GCGCCCAATACGACCA-3'. The cDNA was amplified using the SYBR PCR Master mix and $0.4 \mu \mathrm{L}$ of each primer pair. The amplification was performed with an initial step at $95^{\circ} \mathrm{C}$ for $10 \mathrm{~min}$, and 40 cycles of denaturation at $95^{\circ} \mathrm{C}$ for $15 \mathrm{~s}$, annealing at $60^{\circ} \mathrm{C}$ for $15 \mathrm{~s}$ and extension at $72^{\circ} \mathrm{C}$ for $15 \mathrm{~s}$ for profilin-1 and GAPDH. Under optimised conditions, there was a single melting curve and no primerdimer formation. The copy number for each mRNA was determined using a standard curve generated with external standards of a known copy number. All amplification reactions for each sample were performed in triplicate and the results were expressed as the ratio of profilin-1 to GAPDH mRNA.

\section{Protein preparation and western blotting analysis}

After treatment, the cells were lysed and the protein concentrations were measured using the BCA protein assay. The supernatants were separated using 5\% SDSPAGE for $1.5 \mathrm{~h}$ and transferred onto a polyvinylidene difluoride (PVDF) membrane followed by $2 \mathrm{~h}$ incubation in $5 \%$ non-fat milk in PBST $(0.1 \%$ Tween 20 in PBS) or at $4^{\circ} \mathrm{C}$ overnight. The blot was probed using an antibody against rabbit anti-human-profilin-1 (1:1500) and subsequently incubated with FITC-conjugated secondary rat anti-rabbit IgG protected from light. The signal was detected and ratios of the target protein against $\beta$-actin control were calculated using the Odyssey Infrared Imaging System (LI-COR Biosciences).

\section{Immunofluorescent staining}

The formation of actin stress fibres as an index of endothelial cytoskeletal reorganisation was analysed using fluorescence microscopy. Endothelial cells on coverslip were fixed in $3.7 \%$ formaldehyde for $10 \mathrm{~min}$ at room temperature and extracted with $0.1 \%$ Triton X-100 for 5 min. Each coverslip was incubated with a rabbit antihuman antibody against profilin-1 (1:50) and then incubated with a FITC-conjugated secondary rat anti-rabbit IgG (imaged on the green channel). F-actin in the cytoplasm was stained with phallotoxins (1:200) (imaged on the red channel) in 1\% BSA in TBST for $1 \mathrm{~h}$. The cells were then rinsed four times with PBS to remove excess antibodies, and then labelled with 1\% 4',6-diamidino-2phenylindole (DAPI) in PBS for 5 min to visualise nuclei. Non-immune rabbit IgG and no phallotoxins were used as a negative control in consecutive sections. Microscopy was performed using the IX81 FV1000 laser confocal scanning microscope (Olympus, Japan).

\section{Supernatant ICAM-1, NO, and ADMA detection}

ICAM-1 in supernatants were detected using an ELISA kit. The level of nitric oxide (NO) in the medium was determined indirectly by the content of nitrite and nitrate using the Griess reagent, and the absorbance was determined at $540 \mathrm{~nm}$ using a spectrophotometer. The supernatants were obtained for the measurement of ADMA content using high-performance liquid chromatography (HPLC).

\section{Intracellular ROS detecting}

After the cells of each group were treated, the cell culture medium was removed, and $1 \mathrm{ml} \mathrm{2} 2^{\prime}, 7^{\prime}$-dichlorofluorescin diacetate (DCFH-DA) (1:500 diluted concentration) DMEM was added to cover the cells completely. The cells were cultured for an additional $30 \mathrm{~min}$. After rinsing with serumfree DMEM three times to remove free DCFH-DA, the level of ROS in the HUVEC12 cells was observed using the IX81 FV1000 laser confocal scanning microscope (Olympus, Japan) with an excitation wavelength at $488 \mathrm{~nm}$.

\section{Measurement of PKC activity}

Endothelial cells $\left(5 \times 10^{6}\right.$ to $\left.1 \times 10^{7}\right)$ were extracted according to the manufacturer's protocol. The amount of phosphorylated and nonphosphorylated peptide species was detected using spectrophotometry at a length of $570 \mathrm{~nm}$. The kinase activity was quantified using the PepTag Assay for NonRadioactive Detection of Protein Kinase C.

\section{Measurement of NF-KB activity}

$\mathrm{NF}-\kappa \mathrm{B}$ activity in endothelial cells was detected using an NF-kB-luciferase reporter vector (pGL4.32[luc2P/ NF-kB-RE/Hygro] vector). Briefly, $5 \times 10^{6}$ endothelial cells were transiently co-transfected with $\mathrm{pSV}-\beta$-galactosidase and pGL4.32[luc2P/NF-kB-RE/Hygro] using liposome-mediated transfection at a DNA/lipid ratio of $1 \mu \mathrm{g}$ of each plasmid DNA/2 $\mu \mathrm{l}$ of Lipofectamine 2000. The cells were allowed to recover for $24 \mathrm{~h}$ prior to being 
plated into 24 -well plates at $1 \times 10^{5}$ cells/well and the luciferase activity was measured using a Victor 4 multi-labelled counter and the Luciferase Assay System kit according to manufacturer's instructions. The intensity of the luciferase activity in these cells was normalised against the $\beta$ galactosidase activity, which was used as an internal control. The luciferase activity was expressed as a fold increase.

\section{RNA interference and cell transfection}

To silence profilin-1 gene expression, pLNCX2-siprofilin-1 plasmids were transfected into HUVEC12 cells using Lipofectamine 2000 when the cells were $70 \%$ confluent according to the manufacturer's protocol. The transfection efficiency for each experiment was determined using the percentage of the cells that expressed GFP (green fluorescent protein) under a fluorescent microscope (Nikon, Japan) or by western blotting analysis and real-time PCR.

\section{Statistical analysis}

All data were expressed as the mean \pm SD. The ANOVA test was used to detect differences between groups and the LSD or Dunnett T3 test was used for multiple comparisons. A value of $\mathrm{P}<0.05$ was considered significant.

\section{Results}

The mRNA and protein expression of profilin-1 in endothelial cells

Compared with the control, incubation of endothelial cells with AGEs $(100 \mu \mathrm{g} / \mathrm{ml}, 200 \mu \mathrm{g} / \mathrm{ml}, 400 \mu \mathrm{g} / \mathrm{ml})$ significantly up-regulated the mRNA and protein expression of profilin1 at $4 \mathrm{~h}$ or $24 \mathrm{~h}(P<0.05, P<0.01)$. In addition, treatment with AGEs at a dose of $200 \mu \mathrm{g} / \mathrm{ml}$ had a most robust effect on profilin-1 gene and protein expression (Figure 1A, C), and demonstrated up-regulation of profilin-1 protein expression in a time-dependent manner $(P<0.05, P<0.01$, Figure 1B). Thus, AGEs at a dose of $200 \mu \mathrm{g} / \mathrm{ml}$ for $24 \mathrm{~h}$ was selected for further studies. Pretreatment with DPI (10 $\mu \mathrm{mol} / \mathrm{L})$, GF 109203X $(10 \mu \mathrm{mol} / \mathrm{L})$ and BAY-117082 (5 $\mu \mathrm{mol} / \mathrm{L})$ significantly decreased the up-regulated mRNA and protein expression of profilin-1 mediated by AGEs $(200 \mu \mathrm{g} / \mathrm{ml}, 24 \mathrm{~h})$ (Figure 2).

\section{Endothelial cells abnormalities}

In cultured endothelial cells, treatment with AGEs (200 $\mu \mathrm{g} / \mathrm{ml}, 24 \mathrm{~h}$ ) markedly increased the levels of ICAM-1 and ADMA, and decreased the synthesis of NO, which was

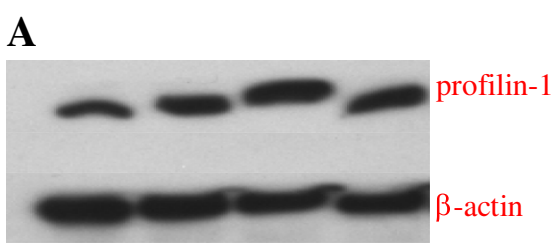

B
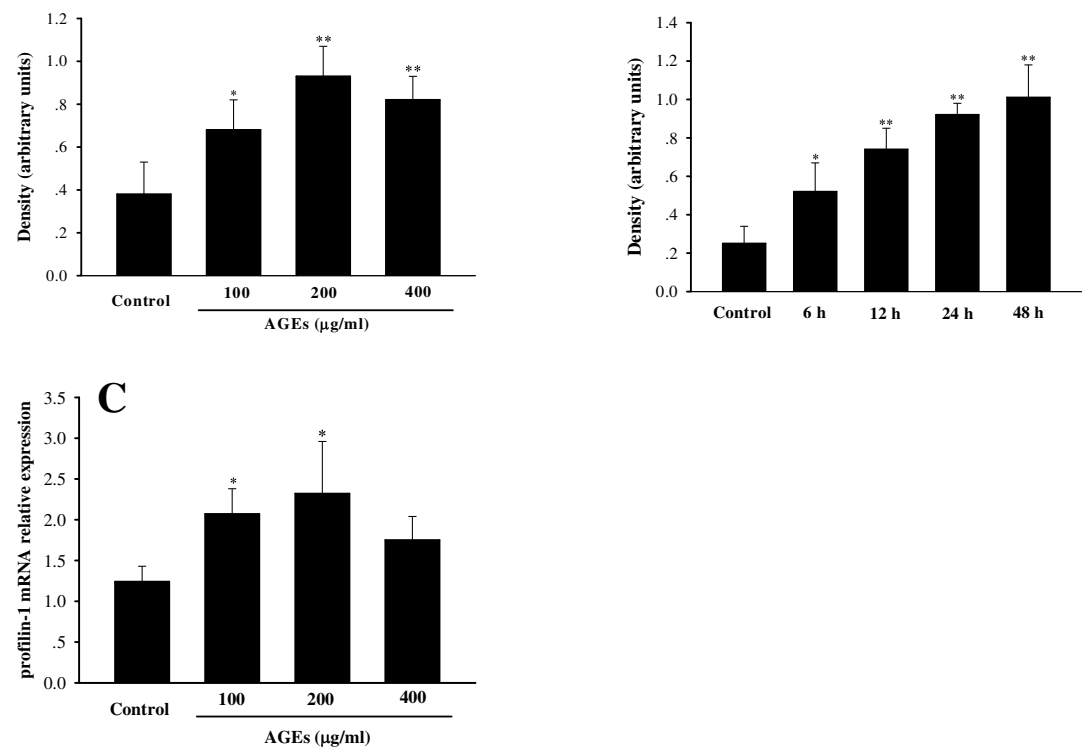

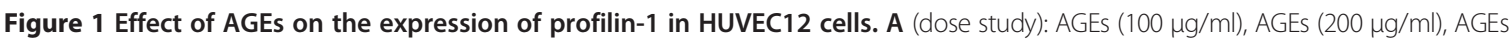
( $400 \mu \mathrm{g} / \mathrm{ml})$ : Endothelial cells were incubated with AGEs $(100 \mu \mathrm{g} / \mathrm{ml}, 200 \mu \mathrm{g} / \mathrm{ml}, 400 \mu \mathrm{g} / \mathrm{ml}$ ) for $24 \mathrm{~h}$. B (time study): $6 \mathrm{~h}, 12 \mathrm{~h}, 24$ h, 48 h: endothelial cells were incubated with $200 \mu \mathrm{g} / \mathrm{ml}$ AGEs for $6 \mathrm{~h}, 12 \mathrm{~h}, 24 \mathrm{~h}, 48 \mathrm{~h}$. C: Effect of AGEs on the mRNA expression of profilin-1: Endothelial cells were incubated with AGEs $(100 \mu \mathrm{g} / \mathrm{ml}, 200 \mu \mathrm{g} / \mathrm{ml}, 400 \mu \mathrm{g} / \mathrm{ml})$ for $4 \mathrm{~h} . \mathrm{n}=3,{ }^{*} \mathrm{P}<0.05 \mathrm{vs}$ control, ${ }^{* *} \mathrm{P}<0.01$ vs control. 
attenuated by pretreatment with DPI, GF 109203X and BAY-117082 $(P<0.05, P<0.01$, Figure 3$)$.

Actin stress fibre formation in endothelial cells during stress is a commonly recognised cytoskeletal response, which indicates reorganisation of the actin cytoskeleton [12]. Thus, we assessed the effects of AGEs on actin cytoskeletal redistribution using fluorescence confocal microscopy, which enables visualisation of profilin-1 and actin filaments. As shown in Figure 4, F-actin was well-distributed at the edge of the cytomembrane in the control group; however, F-actin fibre morphology and distribution showed a marked change after treatment with AGEs. AGEs $(200 \mu \mathrm{g} / \mathrm{ml}, 24 \mathrm{~h})$ induced the appearance of many coarse and short actin stress fibres in the cytoplasm. Immunofluorescent staining showed that the level of green fluorescence in the AGE treatment group was significantly higher than the control group, further supporting that the expression levels of profilin-1 were up-regulated with AGE treatment. Pretreatment with DPI, GF 109203X and BAY-117082 markedly lowered the green fluorescent expression and decreased the formation of actin stress fibre in the cytoplasm.

\section{The protective role of profilin-1 siRNA in AGE-induced endothelial damage}

To confirm the role of profilin-1 in AGE-mediated endothelial injury, we generated specific pLNCX2-siprofilin-1 plasmids to knock down the expression of profilin-1. Pretreatment with $4 \mu \mathrm{g}$ pLNCX2-siprofilin-1 plasmids successfully knocked down the mRNA and protein expression of profilin-1 induced by AGEs in HUVECs $(P<0.01$, Figure 2$)$. Importantly, profilin-1 siRNA blunted the endothelial injury induced by AGEs $(200 \mu \mathrm{g} / \mathrm{ml}, 24 \mathrm{~h})$ as shown by the significant decrease in the levels of ICAM-1 and ADMA, and the increase in $\mathrm{NO}$ production $(\mathrm{P}<0.05, \mathrm{P}<0.01$, Figure 3). Immunofluorescent staining showed that silencing profilin-1 gene expression markedly decreased the expression of profilin-1 (red fluorescence, Figure 5B) in the cytoplasm and improved F-actin redistribution (red fluorescence) in the presence of AGEs (Figure 5C). Overall, these results confirmed that AGEs induced actin cytoskeletal reorganisation and redistribution via increasing the levels of profilin-1 in the cytoplasm.

\section{Involvement of PKC and the NF-KB pathway in AGE-induced endothelial injury}

To explore the potential signal pathway involved in AGEinduced endothelial injury, DPI (antioxidant), GF 109203X (PKC inhibitor) and BAY 117082 (NF- $\mathrm{kB}$ inhibitor) were incubated for $1 \mathrm{~h}$ prior to AGEs stimulation. These results showed that antioxidants or blockade of the PKC or NF- $\mathrm{kB}$ pathways significantly improved the endothelial abnormalities induced by AGEs, concomitantly with downregulation of profilin-1 expression (Figures 2, 3, 4). In

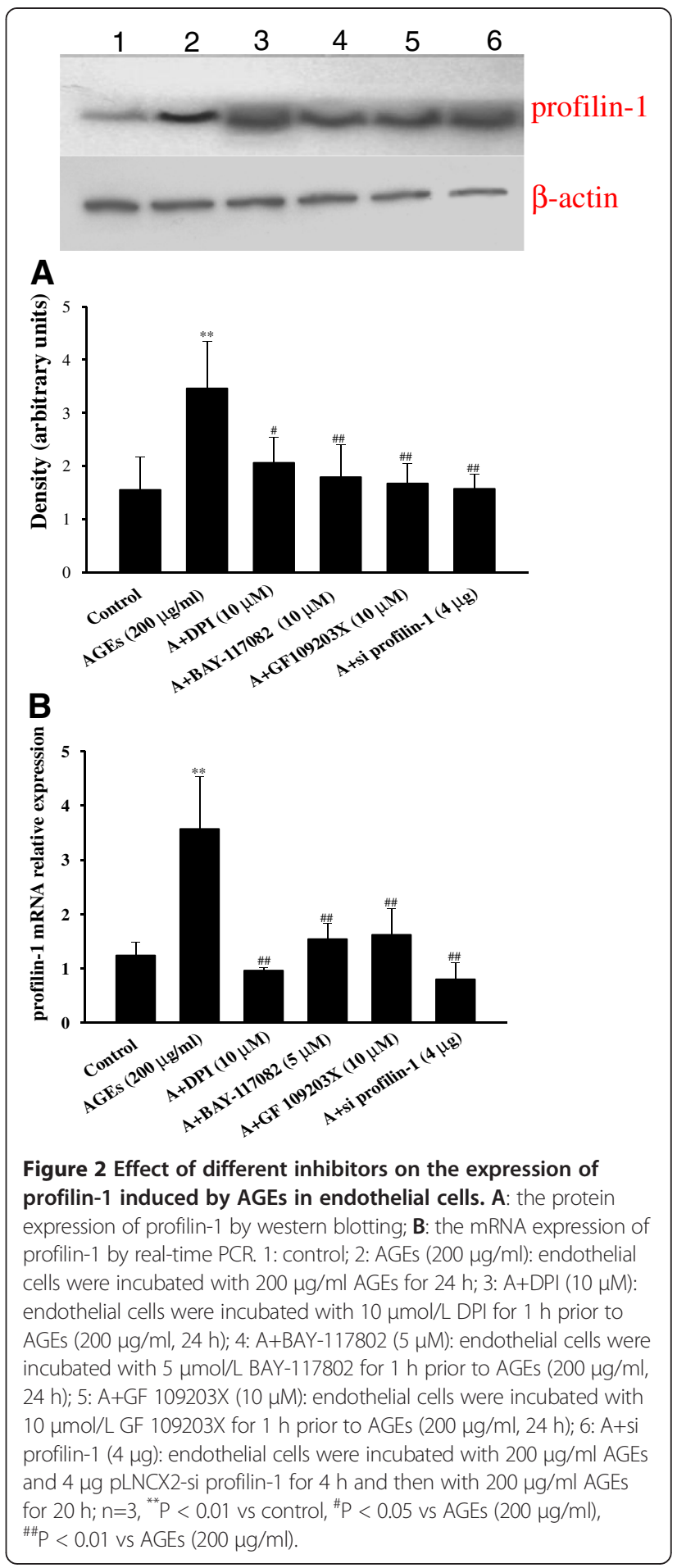

addition, DPI markedly decreased the elevated levels of ROS induced by AGEs (200 $\mu \mathrm{g} / \mathrm{ml}, 24 \mathrm{~h})$; however, GF 109203X and BAY-117082 showed no significant effect on ROS formation induced by AGEs (Figure 6). Because marked green fluorescence showed mutual interference, the intracellular ROS levels were not determined in the interference study. Expectedly, DPI and GF 

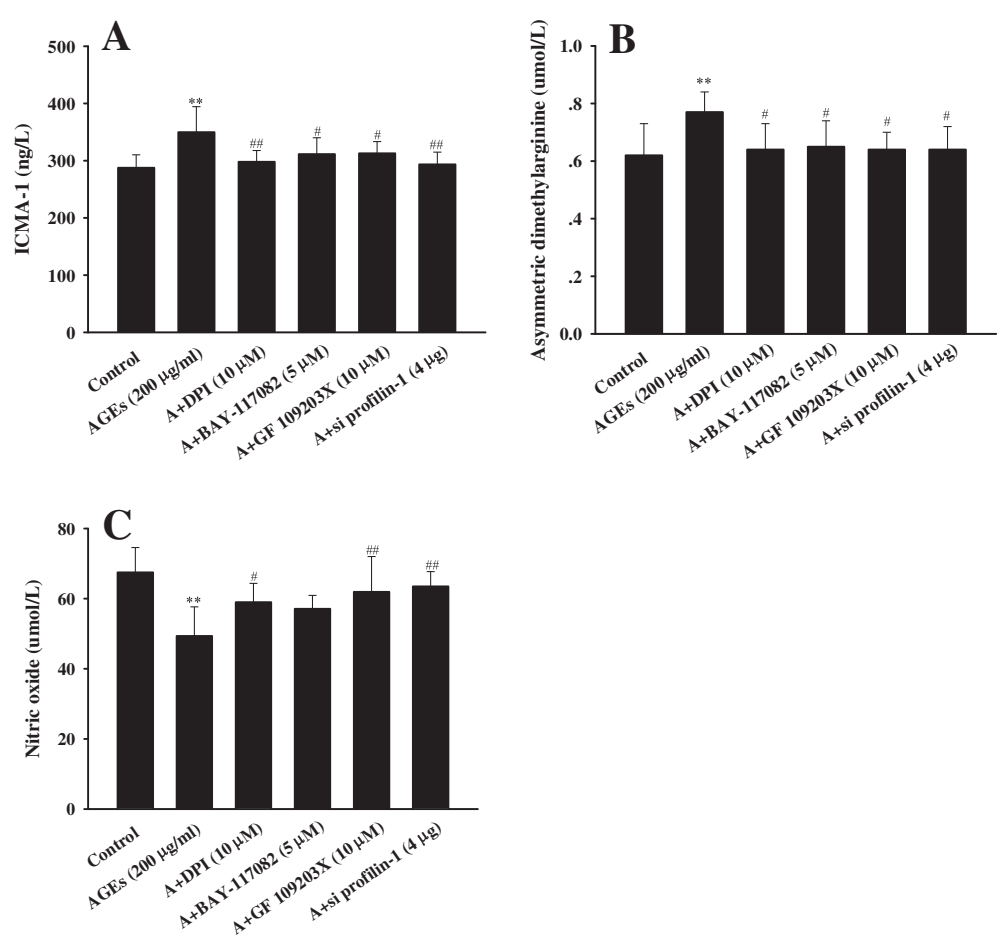

Figure 3 Effect of different inhibitors on the levels of cytokines induced by AGEs in endothelial cells. A: the levels of ICAM-1; B: the levels of ADMA; C: the levels of NO. $n=6,{ }^{* *} \mathrm{P}<0.01$ vs control, ${ }^{\#} \mathrm{P}<0.05$ vs AGEs $(200 \mu \mathrm{g} / \mathrm{ml})$, ${ }^{\# \#} \mathrm{P}<0.01$ vs AGEs $(200 \mu \mathrm{g} / \mathrm{ml})$.

109203X significantly inhibited the activity of PKC, and DPI and BAY-117082 markedly prevented the activation of NF-kB $(P<0.05, P<0.01)$. However, GF 109203X had no effect on the activity of NF- $\mathrm{B}$, and BAY-117082 had no effect on the activity of PKC $(P>0.05)$. Blockade of profilin-1 expression also had no effect on the activation of PKC and NF-кB induced by AGEs (Figure 7). These results suggested that AGEs mediated endothelial abnormalities via the excess formation of ROS and subsequent activation of the PKC and NF- $\mathrm{BB}$ pathways. Furthermore, profilin-1 is the ultimate and common downstream effector in endothelial injury.

\section{Discussion}

The main findings of the present study are as follows: (1) AGEs induced endothelial injury as shown by increasing ADMA and ICAM levels and decreasing the synthesis of NO, which occured concomitantly with the up-regulated expression of profilin-1 and the rearrangement and redistribution of F-actin. This was attenuated by treatment with the antioxidant DPI, PKC inhibitor GF109203X or NF-kB inhibitor BAY117082; (2) Knockdown of profilin-1 gene expression attenuated AGE-induced endothelial abnormalities; (3) AGEs up-regulated the expression of profilin-1 via the excess production of ROS and subsequent activation of $\mathrm{PKC}$ and NF-kB. Taken together, these findings suggested, for the first time, that profilin-1 plays an important role in endothelial injury induced by AGEs, which may contribute to macrovascular complications in DM.

There is accumulating evidence that AGEs formation due to chronic hyperglycaemia has a chemical, cellular and tissue effect in metabolic memory. AGEs have been previously associated with the development of diabetesrelated macrovascular and microvascular complications [13-15]. It has been recently reported that dietary AGEs in diabetic patients or intraperitoneal injection of AGEs in rats caused an impairment in the vascular endothelium [16-18], which was prevented by treatment with the AGE inhibitor benfotiamine [16]. Accumulation of AGEs in the vasculature initiated a series of morphological and functional changes in endothelial cells and induced an increase in endothelial permeability and cell apoptosis. Moreover, it also promoted endothelial (progenitor) cell migration, adhesion and focal contact formation, concomitantly with the decreased synthesis of NO and the activity of superoxide dismutase (SOD) $[10,18,19]$. It is well-known that the vascular endothelium is an important target of hyperglycaemic damage and an increase in endothelial permeability to macromolecules results in vascular dysfunction that is associated with several pathological states, including diabetes. Previous studies have demonstrated that the elevated ADMA levels connected with the uncoupling of NO synthesis contributing to endothelial dysfunction were 

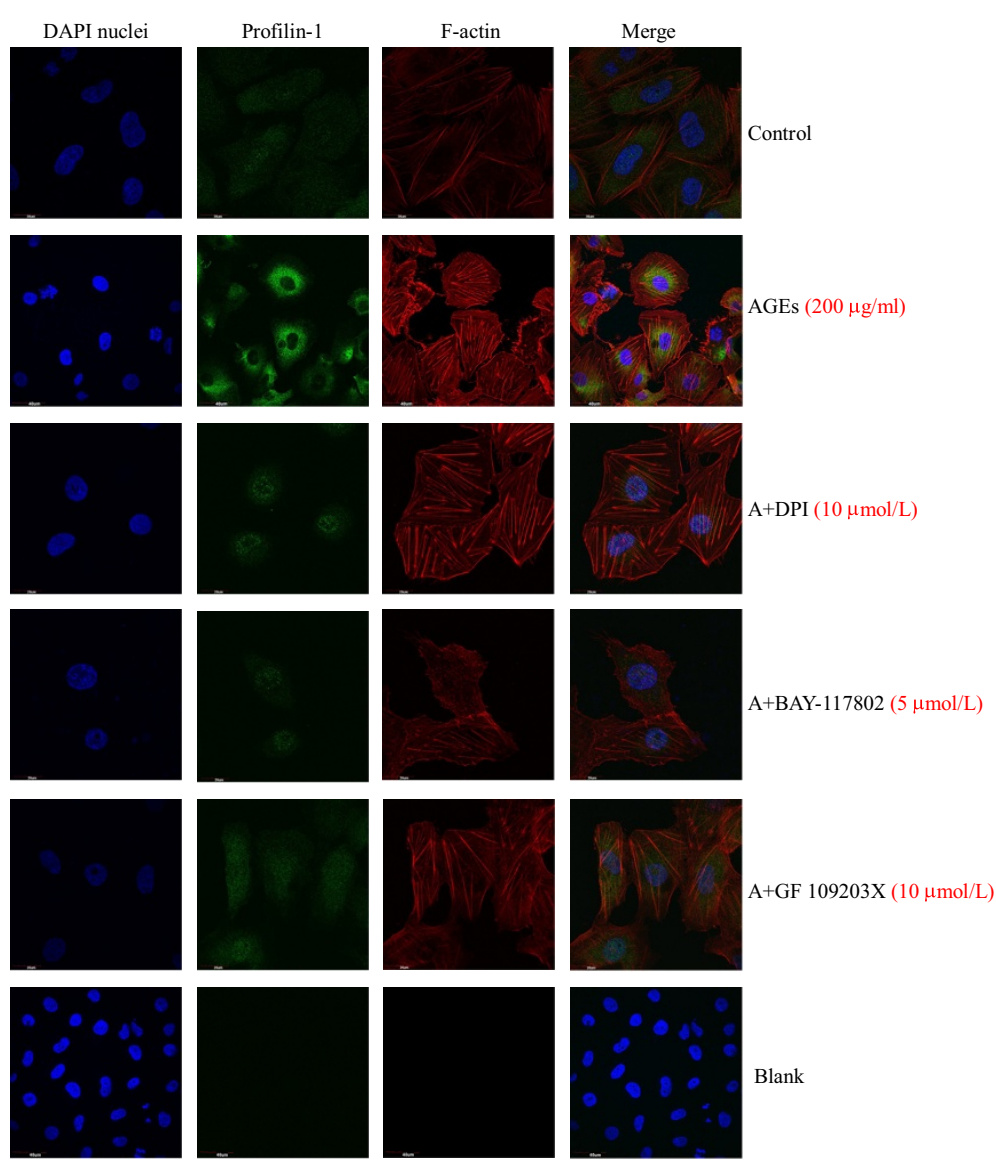

Figure 4 Effect of different inhibitors on the expression of profilin-1 and the distribution of F-actin induced by AGEs in endothelial cells. $\mathrm{n}=3$. Immunofluorescence staining for profilin-1 (green), F-actin (red) and 4',6-diamidino-2-phenylindole (DAPI) nuclei (blue). Non-immune rabbit lgG was used as blank.

associated with future cardiovascular events in diabetic patients [20]. Indeed, ADMA has been recognised as a marker of endothelial dysfunction and as a risk factor of cardiovascular diseases [21,22]. It was recently reported that AGEs markedly increased ADMA levels in tubular and endothelial cells via the stimulation of local ROS production [23,24]. A great deal of studies have demonstrated that circulating level of SICAM-1 is considered to be one important marker of endothelial dysfunction and it was reported that the endothelial dysfunction marked by impaired acetylcholine-induced endothelium-dependent relaxation of aortic rings and elevated levels of sICAM-1 were present in streptozocin-induced diabetic rats [25]. In the present study, we explored the effect of AGEs on the adverse actions in cultured endothelial cells. Consistent with previous studies, incubation with AGEs markedly reduced the synthesis of NO, increased the levels of ADMA, ICAM-1 and intracellular ROS, which was attenuated by pretreatment with the antioxidant DPI, suggesting that exogenous AGEs directly induced endothelial injury via the overproduction of ROS.
Profilin-1, an intracellular actin-binding protein, achieves its function via the regulation of the size, localization and dynamics of unpolymerised actin in cells contributing to endothelial cell contraction and vascular hyperpermeability. Previous studies have demonstrated that cardiovascular risk factors, such as homocysteine, LDL, ox-LDL, and oxidised cholesterol can up-regulated the expression of profilin-1 in cultured endothelial cells, resulting in cytoskeletal structural remodelling $[8,26,27]$. Recently, it has been reported that overexpression of profilin-1 up-regulated the expression of ICAM-1, increased endothelial cell permeability, induced endothelial cell apoptosis and promoted endothelial cell migration, adhesion and focal contact formation $[7,9]$ and silencing profilin-1 gene expression provided significant protection on endothelial cells [28]. In vivo studies have further demonstrated that profilin-1 expression was upregulated in endothelial cells and macrophages in atherosclerotic lesions in ApoE null mice, and in the aorta of diabetic rats, and the serum levels of profilin-1 were significantly elevated in patients with severe AS $[8,29]$. In contrast, attenuated expression of profilin-1 conferred 


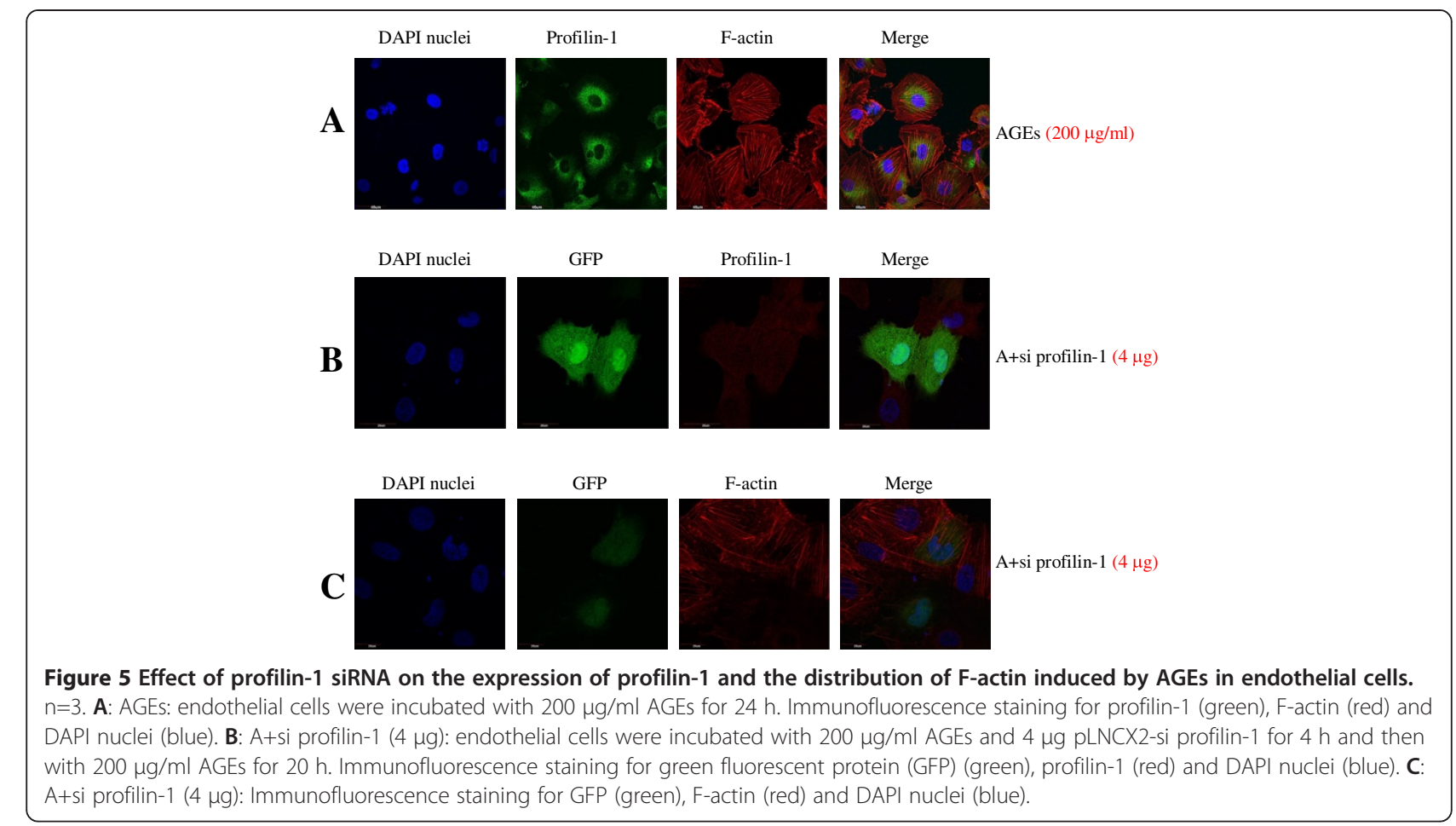

protection against AS in LDL receptor null mice [8]. Recently, Romeo et al. reported that high-fat diet (HFD) upregulated the expression of profilin-1 in both stromal vascular cells and adipocytes of white adipose tissue (WAT) and pfn heterozygote mice (PfnHet) displayed near normalization of HFD-induced glucose intolerance and the release of pro-inflammatory cytokines, suggesting that profilin-1 plays an bran-new role in modulation of immune homeostasis within the WAT microenvironment [30]. Because endothelial damage stimulated by profilin-1 in diabetes is surprisingly similar with that in lipid oxidation, it is conceivable that profilin-1 may function in a common pathway that induces vascular endothelial injuries in vascular lesions. It has been previously reported that incubation with AGEs caused a significant increase in the permeability of endothelial cell monolayers via significant disorganisation

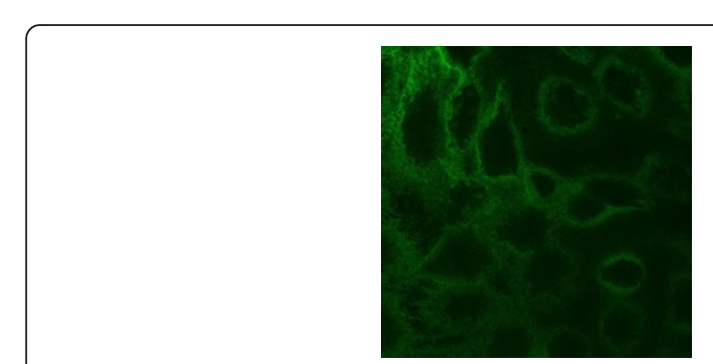

control
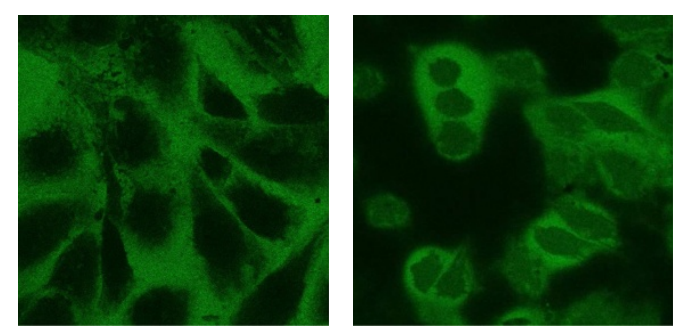

A+BAY-117802 (5 $\mu \mathrm{M})$ A+GF 109203X $(10 \mu \mathrm{M})$

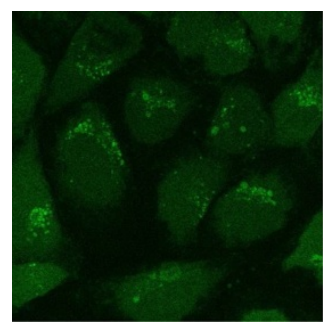

A+DPI $(10 \mu \mathrm{M})$

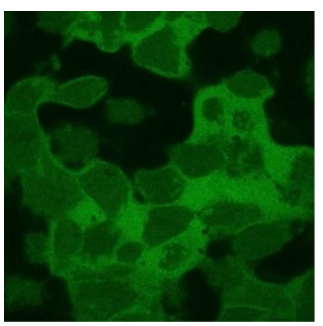

AGEs $(200 \mu \mathrm{g} / \mathrm{ml})$

Figure 6 Effect of different inhibitors on the levels of intracellular ROS mediated by AGEs in endothelial cells. $n=3$. 

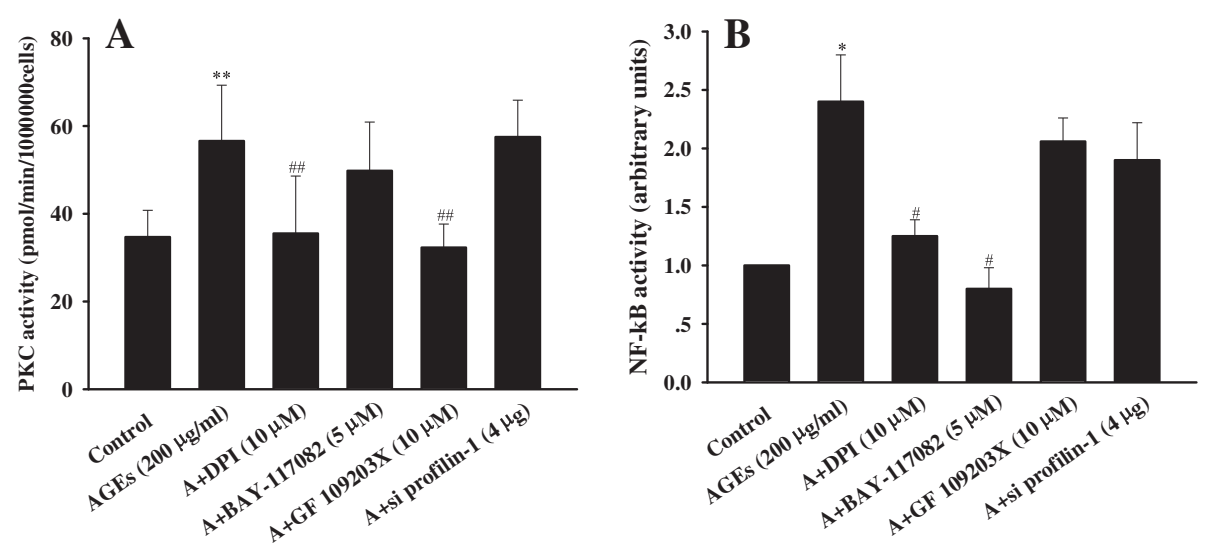

Figure 7 Effect of different inhibitors on the signaling pathways mediated by AGEs in endothelial cells. A: the activity of PKC; B: the activity of NF-қB. $n=4,{ }^{*} P<0.05$ vs control, ${ }^{* *} P<0.01$ vs control, ${ }^{\#} P<0.05$ vs AGEs $(200 \mu \mathrm{g} / \mathrm{ml}),{ }^{\# \#} \mathrm{P}<0.01$ vs AGEs $(200 \mu \mathrm{g} / \mathrm{ml})$.

of the F-actin cytoskeleton and disruption of tight and adherent junctions [10,11,31-33]. However, detailed studies on the role of profilin-1 in AGE-induced adverse actions in vascular endothelial cells are lacking. In addition, the protection offered by specifically blocking the expression of profilin-1 against AGE-induced endothelial alterations has not yet been reported. This study found that treatment with AGEs markedly up-regulated the mRNA and protein expression of profilin-1, which was accompanied by the rearrangement and redistribution of the cytoskeleton. However, silencing profilin-1 expression significantly attenuated endothelial injury by increasing the synthesis of $\mathrm{NO}$ and decreasing the levels of ADMA and ICAM-1. This occurred concomitantly with the attenuation of the cytoskeletal rearrangement. To the best of our knowledge, this is the first report demonstrating that AGEs up-regulated the expression of profilin-1 in vascular endothelial cells and the silencing of profilin-1 expression induced endothelial protection against AGEs effects.

It is well known that AGEs mediate metabolic memory via its receptor RAGE to produce excess ROS, and subsequently activates $\mathrm{PKC}$ and $\mathrm{NF}-\mathrm{kB}$ signalling via intracellular signalling cascades. This process induces the expression of a variety of diabetes-related genes $[1,5]$. Normalisation of the levels of mitochondrial ROS prevented glucose-induced activation of PKC and NF$\mathrm{KB}$, the formation of AGEs and sorbitol accumulation [34]. It has been previously reported that AGEs induced PKC- $\beta$ translocation, extracellular signal-regulated protein kinase $1 / 2$ and NF- $\mathrm{KB}$ activation in bovine retinal endothelial cell, and pharmacological inhibition of these signalling pathways and antioxidants abolished the effects mediated by AGEs [35]. Substantial evidence indicates that activation of PKC and NF-KB is a key biochemical event implicated in the development of diabetic vascular complications [36,37]. To elucidate the potential signal pathway involved in endothelial injury induced by AGEs, antioxidant DPI, PKC inhibitor (GF 109203X) and NF-kB inhibitor (BAY-117082) were employed. These results showed that pretreatment with DPI, GF 109203X and BAY-117082 attenuated endothelial injury, down-regulated the elevated expression of profilin-1 and ameliorated the actin cytoskeleton rearrangement and redistribution induced by AGEs. Antioxidant DPI markedly inhibited the formation of ROS and activation of PKC and NF- $\mathrm{KB}$ signalling by AGEs. However, blockade of the PKC and NF-kB pathways had no significant effect on the overproduction of ROS. It was previously reported that shear stress increased the production of ROS and the activity of PKC in aortic endothelial cells, and the increase in ROS production was unaffected by GF109203X, whereas the activation of PKC was reduced by antioxidant $\mathrm{N}$-acetyl-L-cysteine (NAC) [38]. Thus we concluded that oxidative stress triggered by AGEs subsequently activated downstream NF- $\mathrm{kB}$ and PKC signalling pathways. Interestingly, silencing of profilin-1 gene expression induced endothelial protection, but did not affect the activity of NF-KB and PKC. Thus, it can be inferred that AGEs up-regulated the expression of profilin-1 and caused damage in endothelial cells via the production of excess ROS, thereby activating NF- $\mathrm{kB}$ and PKC signalling pathways. Thus, we speculated that profilin-1 functions as an ultimate and common cellular channel in endothelial abnormalities mediated by AGEs.

In conclusion, the present study suggests, for the first time, that profilin-1 is a downstream molecule that mediates endothelial injury induced by AGEs via the ROS/ PKC or ROS/NF- $\mathrm{kB}$ signalling pathways, and blockade of profilin-1-mediated biological effects may help to prevent endothelial injuries and vascular lesions in diabetes. Taken together, these findings set the stage for a prominent role of profilin-1 as a modulator of the actin cytoskeleton, which may underlie the pathology of vascular diseases, including diabetes. 


\section{Abbreviations}

ADMA: Asymmetric dimethylarginine; AGE: Advanced glycation end products: AGE-BSA: AGE-bovine serum albumin; AS: Atherosclerosis; DAPI: 4',6-diamidino2-phenylindole; DCFH-DA: 2', 7'-dichlorofluorescin diacetate; DM: Diabetes mellitus; DMEM: Dulbecco's modified eagle's medium; DPI: Diphenyliodonium; FBS: Foetal bovine serum; GFP: Green fluorescent protein; HFD: High-fat diet; HPLC: High-performance liquid chromatography; HUVEC: Human umbilical vein endothelial cells; ICAM-1: Intercellular adhesion molecule-1; LDL: Low density lipoprotein; NAC: N-acetyl-L-cysteine; NF-kB: Nuclear factor kappa B; NO: Nitric oxide; PBS: Phosphate-buffered saline; PfnHet: Pfn heterozygote mice; PKC: Protein kinase C; PVDF: Polyvinylidene difluoride; RAGE: Receptor for advanced glycation end products; ROS: Reactive oxidative species; SOD: Superoxide dismutase; WAT: White adipose tissue.

\section{Competing interests}

The authors declare that they have no competing interests.

\section{Authors' contributions}

MC conceived the study, arranged the collaboration, initiated the manuscript, edited and compiled the final version for submission. ZL and QZ performed laboratory work and data analysis. TY and XX participated in its design and coordination. All authors read and approved the final manuscript.

\section{Acknowledgments}

The work was supported by the National Nature Science Foundation of China (No. 81000140) and Young Teacher Promotion Plan of Central South University (No. 1801725).

\section{Author details}

${ }^{1}$ Department of Geriatric Medicine, Xiang-Ya Hospital, Central South University, Xiang-Ya Road 87\#, Changsha, Hunan 410008, China. ${ }^{2}$ Department of Cardiology, the First People's Hospital of Chenzhou, Chenzhou, Hunan 423000, China. ${ }^{3}$ Department of Cardiology, Xiang-Ya Hospital, Central South University, Changsha, Hunan 410008, China.

Received: 11 August 2013 Accepted: 28 September 2013 Published: 4 October 2013

\section{References}

1. Ceriello A: The emerging challenge in diabetes: the "metabolic memory". Vascul Pharmacol 2012, 57:133-138.

2. Genuth S, Sun W, Cleary P, Sell DR, Dahms W, Malone J, Sivitz W, Monnier VM, DCCT Skin Collagen Ancillary Study Group: Glycation and carboxymethyllysine levels in skin collagen predict the risk of future 10year progression of diabetic retinopathy and nephropathy in the diabetes control and complications trial and epidemiology of diabetes interventions and complications participants with type 1 diabetes. Diabetes 2005, 54:3103-3111.

3. Kiuchi K, Nejima J, Takano T, Ohta M, Hashimoto H: Increased serum concentrations of advanced glycation end products: a marker of coronary artery disease activity in type 2 diabetic patients. Heart 2001, 85:87-91.

4. Ceriello A, Ihnat MA, Thorpe JE: The "metabolic memory": is more than just tight glucose control necessary to prevent diabetic complications? J Clin Endocrinol Metab 2009, 94:410-415.

5. Guo ZJ, Niu HX, Hou FF, Zhang L, Fu N, Nagai R, Lu X, Chen BH, Shan YX, Tian JW, Nagaraj RH, Xie D, Zhang X: Advanced oxidation protein products activate vascular endothelial cells via a RAGE-mediated signaling pathway. Antioxid Redox Signal 2008, 10:1699-1712.

6. Bogatcheva NV, Garcia JG, Verin AD: Molecular mechanisms of therombininduced endothelial cell permeability. Biochemistry (MOSC) 2002, 67:75-84

7. Romeo G, Frangioni JV, Kazlauskas A: Profilin acts downstream of LDL to mediate diabetic endothelial cell dysfunction. FASEB J 2004, 18:725-727.

8. Romeo GR, Moulton KS, Kazlauskas A: Attenuated expression of profilin-1 confers protection from atherosclerosis in the LDL receptor null mouse. Circ Res 2007, 101:357-367.

9. Romeo GR, Kazlauskas A: Oxysterol and diabetes activate STAT3, and control endothelial expression of profilin-1 via OSBP1. J Biol Chem 2008, 283:9595-9605.
10. Wang J, Liu H, Chen B, Li Q, Huang X, Wang L, Guo X, Huang Q: RhoA/ ROCK-dependent moesin phosphorylation regulates AGE-induced endothelial cellular response. Cardiovasc Diabetol 2012, 11:7.

11. Guo XH, Huang QB, Chen B, Wang SY, Li Q, Zhu YJ, Hou FF, Fu N, Brunk UT, Zhao M: Advanced glycation end products induce actin rearrangement and subsequent hyperpermeability of endothelial cells. APMIS 2006, 114:874-883.

12. Vandenbroucke $E$, Mehta $D$, Minshall $R$, Malik AB: Regulation of endothelial junctional permeability. Ann N Y Acad Sci 2008, 1123:134-145.

13. Basta G, Schmidt AM, De Caterina R: Advanced glycation end products and vascular inflammation: implications for accelerated atherosclerosis in diabetes. Cardiovasc Res 2004, 63:582-592.

14. Rojas A, Morales MA: Advanced glycation and endothelial functions: a link towards vascular complications in diabetes. Life Sci 2004, 76:715-730.

15. Seki N, Hashimoto N, Taira M, Yagi S, Yoshida Y, Ishikawa K, Suzuki Y, Sano H, Horiuchi S, Yoshida S, Sakurai K, Yagui K, Makino H, Saito Y: Regulation of Src homology 2-containing protein tyrosine phosphatase by advanced glycation end products: the role on atherosclerosis in diabetes. Metabolism 2007, 56:1591-1598.

16. Stirban A, Negrean M, Stratmann B, Gawlowski T, Horstmann T, Götting C, Kleesiek K, Mueller-Roesel M, Koschinsky T, Uribarri J, Vlassara H, Tschoepe D: Benfotiamine prevents macro- and microvascular endothelial dysfunction and oxidative stress following a meal rich in advanced glycation end products in individuals with type 2 diabetes. Diabetes Care 2006, 29:2064-2071.

17. Negrean M, Stirban A, Stratmann B, Gawlowski T, Horstmann T, Götting C, Kleesiek K, Mueller-Roesel M, Koschinsky T, Uribarri J, Vlassara H, Tschoepe D: Effects of low- and high-advanced glycation endproduct meals on macro- and microvascular endothelial function and oxidative stress in patients with type 2 diabetes mellitus. Am J Clin Nutr 2007, 85:1236-1243.

18. Soro-Paavonen A, Zhang WZ, Venardos K, Coughlan MT, Harris E, Tong DC, Brasacchio D, Paavonen K, Chin-Dusting J, Cooper ME, Kaye D, Thomas MC, Forbes JM: Advanced glycation end-products induce vascular dysfunction via resistance to nitric oxide and suppression of endothelial nitric oxide synthase. J Hypertens 2010, 28:780-788.

19. Li H, Zhang X, Guan X, Cui X, Wang Y, Chu H, Cheng M: Advanced glycation end products impair the migration, adhesion and secretion potentials of late endothelial progenitor cells. Cardiovasc Diabetol 2012, 11:46.

20. Yamagishi S, Ueda S, Nakamura K, Matsui T, Okuda S: Role of asymmetric dimethylarginine (ADMA) in diabetic vascular complications. Curr Pharm Des 2008, 14:2613-2618.

21. Tarnow L, Hovind P, Teerlink T, Stehouwer CD, Parving HH: Elevated plasma asymmetric dimethylarginine as a marker of cardiovascular morbidity in early diabetic nephropathy in type 1 diabetes. Diabetes Care 2004, 27:765-769.

22. Sibal $L$, Agarwal SC, Home PD, Boger RH: The role of asymmetric dimethylarginine (ADMA) in endothelial dysfunction and cardiovascular disease. Curr Cardiol Rev 2010, 6:82-90.

23. Ojima A, Ishibashi Y, Matsui T, Maeda S, Nishino Y, Takeuchi M, Fukami K, Yamagishi S: Glucagon-like peptide-1 receptor agonist inhibits asymmetric dimethylarginine generation in the kidney of streptozotocin-induced diabetic rats by blocking advanced glycation end product-induced protein arginine methyltranferase-1 expression. Am J Pathol 2013, 182:132-141.

24. Ando R, Ueda S, Yamagishi S, Miyazaki H, Kaida Y, Kaifu K, Yokoro M, Nakayama Y, Obara N, Fukami K, Takeuchi M, Okuda S: Involvement of advanced glycation end product-induced asymmetric dimethylarginine generation in endothelial dysfunction. Diab Vasc Dis Res 2013, 10:436-441.

25. Wang LJ, Yu YH, Zhang LG, Wang Y, Niu N, Li Q, Guo LM: Taurine rescues vascular endothelial dysfunction in streptozocin-induced diabetic rats: correlated with downregulation of LOX-1 and ICAM-1 expression on aortas. Eur J Pharmacol 2008, 597:75-80.

26. Moldovan NI, Milliken EE, Irani K, Chen J, Sohn RH, Finkel T, GoldschmidtClermont PJ: Regulation of endothelial cell adhesion by profilin. Curr Biol 1997, 7:24-30.

27. Dardik R, Savion N, Gal N, Varon D: Flow conditions modulate homocysteine induced changes in the expression of endothelial cell genes associated with cell-cell interaction and cytoskeletal rearrangement. Thromb Haemost 2002, 88:1047-1053.

28. Ding Z, Lambrechts A, Parepally M, Roy P: Silencing profilin- 1 inhibits endothelial cell proliferation, migration and cord morphogenesis. J Cell Sci 2006, 119:4127-4137. 
29. Caglayan E, Romeo GR, Kappert K, Odenthal M, Südkamp M, Body SC, Shernan SK, Hackbusch D, Vantler M, Kazlauskas A, Rosenkranz S: Profilin-1 is expressed in human atherosclerotic plaques and induces atherogenic effects on vascular smooth muscle cells. PLoS One 2010, 5:e13608.

30. Romeo GR, Pae M, Lee J, Shoelson SE: Profilin-1 haploinsufficiency protects against obesity-associated glucose intolerance and preserves adipose tissue immune homeostasis. Diabetes 2013. Epub ahead of print.

31. Hirose A, Tanikawa T, Mori H, Okada Y, Tanaka Y: Advanced glycation end products increase endothelial permeability through the RAGE/Rho signaling pathway. FEBS Lett 2010, 584:61-66.

32. Wang Z, Guo X, Liu X, Wang J, Wang L, Huang Q: The morphological changes of vascular endothelial cadherin in human umbilical vein endothelial cells induced by advanced glycation end products. Chin $J$ Arterioscler 2008, 16:505-509.

33. Otero K, Martínez F, Beltrán A, González D, Herrera B, Quintero G, Delgado $R$, Rojas A: Albumin-derived advanced glycation end-products trigger the disruption of the vascular endothelial cadherin complex in cultured human and murine endothelial cells. Biochem J 2001, 359:567-574.

34. Nishikawa T, Edelstein D, Du XL, Yamagishi S, Matsumura T, Kaneda Y, Yorek MA, Beebe D, Oates PJ, Hammes HP, Giardino I, Brownlee M: Normalizing mitochondrial superoxide production blocks three pathways of hyperglycaemic damage. Nature 2000, 404:787-790

35. Mamputu JC, Renier G: Signalling pathways involved in retinal endothelial cell proliferation induced by advanced glycation end products: inhibitory effect of gliclazide. Diabetes Obes Metab 2004, 6:95-103.

36. Rosen P, Nawroth PP, King G, Moller W, Tritschler HJ, Packer L: The role of oxidative stress in the onset and progression of diabetes and its complications: a summary of a congress series sponsored by the UNESCOMCBN, the American diabetes association and the German diabetes society. Diabetes Metab Res Rev 2001, 17:189-212.

37. Romeo G, Liu WH, Asnaghi V, Kern TS, Lorenzi M: Activation of nuclear factor-kappaB induced by diabetes and high glucose regulates a proapoptotic program in retinal pericytes. Diabetes 2002, 51:2241-2248.

38. Niwa K, Sakai J, Karino T, Aonuma H, Watanabe T, Ohyama T, Inanami O, Kuwabara M: Reactive oxygen species mediate shear stress-induced fluidphase endocytosis in vascular endothelial cells. Free Radic Res 2006, 40:167-174.

doi:10.1186/1475-2840-12-141

Cite this article as: Li et al:: The role of profilin-1 in endothelial cell injury induced by advanced glycation end products (AGEs).

Cardiovascular Diabetology 2013 12:141.

\section{Submit your next manuscript to BioMed Central and take full advantage of:}

- Convenient online submission

- Thorough peer review

- No space constraints or color figure charges

- Immediate publication on acceptance

- Inclusion in PubMed, CAS, Scopus and Google Scholar

- Research which is freely available for redistribution 